\section{IMPLANTAÇÃO DA PUERICULTURA E DESAFIOS DO CUIDADO NA ESTRATÉGIA SAÚDE DA FAMÍLIA EM UM MUNICÍPIO DO ESTADO DO CEARÁ}

\author{
Implementation of childcare and care challenges in the Family \\ Health Strategy in a city of the Ceará state
}

\author{
Implantación de la puericultura y los desafios del cuidado \\ de la Estrategia de Salud de la Familia de un municipio del \\ Estado de Ceará
}

\section{RESUMO}

Objetivo: Relatar a experiência da implantação da puericultura e desafios do cuidado na Estratégia Saúde da Família em um município do Estado do Ceará. Síntese dos dados: Trata-se de um estudo de abordagem qualitativa, com caráter descritivo, do tipo relato de experiência, desenvolvido no contexto de uma das Unidades Básicas de Saúde do município de Aracati, Ceará. A sistematização do relato se deu a partir dos registros realizados pela enfermeira residente em um diário de campo, no período compreendido entre os meses de junho de 2013 e abril de 2015. Com a implantação da consulta em puericultura, pode-se perceber a importância de se realizar um acompanhamento sistemático desde o pré-natal, perpassando o momento do puerpério e alcançando o foco principal deste estudo, a saúde da criança. Destaca-se a contribuição do Núcleo de Apoio à Saúde da Família nas consultas e o uso das tecnologias simples favorecendo a interação entre profissionais, comunidade e serviços de saúde. Além disso, algumas fragilidades do processo de trabalho precisaram ser superadas, tais como: obstáculos estruturais, falta de compromisso de alguns profissionais, divergências de condutas, profissionais desmotivados devido à sobrecarga de trabalho e poucos cursos ofertados para qualificação da equipe de saúde. Conclusão: A tarefa não é fácil, os desafios são contínuos, mas a recompensa consegue ser maior e mais gratificante do que qualquer dificuldade vivenciada. Espera-se que este relato possa promover reflexões críticas acerca do trabalho que se tem produzido junto à Estratégia Saúde da Família.

Descritores: Atenção Primária à Saúde; Saúde da Criança; Enfermagem Pediátrica; Comunicação Interdisciplinar; Educação em Saúde.

\section{ABSTRACT}

Objective: To report the experience of childcare implementation and care challenges in the Family Health Strategy in a city of the Ceará state. Data synthesis: Experience report study with a descriptive, qualitative approach, developed within one the Primary Health Care Units in the city of Aracati, Ceará. The report systematization was carried out from the records made in a field diary by a nurse resident, in the period between the months of June 2013 and April 2015. With the implementation of the childcare consultation, one can realize the importance of conducting a systematic follow-up since the prenatal period, including the puerperium and reaching the main focus of this study, the child's health. It highlights the contribution of the Family Health Support Center in the consultations and the use of simple technologies favoring the interaction between professionals, community and health services. Additionally, some weaknesses of the work process had to be overcome, such as: structural barriers; lack of commitment on the part of some professionals; divergences of conducts; unmotivated professionals due to work overload and shortage of training courses offered for qualification of the health team. Conclusion: The task is not easy, the challenges are ongoing, but the benefits can be greater and more rewarding than any experienced difficulty.
Descrição ou avaliação de experiências

\author{
Delane Giffoni Soares ${ }^{(1,2)}$ \\ Mara Cynthia Ximenes \\ Pinheiro $^{(1,3)}$ \\ Danielly Maia de Queiroz ${ }^{(1,2)}$ \\ Dharlene Giffoni Soares ${ }^{(1)}$
}

1) Universidade Estadual do Ceará - UECE - Fortaleza (CE) - Brasil

2) Residência Multiprofissional em Saúde da Família e Comunidade pela Escola de Saúde Pública do Ceará (ESP-CE) Fortaleza (CE) - Brasil

3) Estratégia Saúde da Família da Secretaria de Saúde de Fortaleza - Fortaleza (CE) Brasil

4) Universidade de Fortaleza - UNIFOR Fortaleza (CE) - Brasil
Recebido em: 09/06/2015 Revisado em: 05/11/2015 Aceito em: 19/03/2016 
It is hoped that this report can promote critical thinking about the work that has been produced within the Family Health Strategy.

Descriptors: Primary Health Care; Child Health; Pediatric Nursing; Interdisciplinary Communication; Health Education.

\section{RESUMEN}

Objetivo: Relatar la experiencia de implantación de la puericultura y los desafios del cuidado de la Estrategia de Salud de la Familia de un municipio del Estado de Ceará. Síntesis de los datos: Se trata de un estudio de abordaje cualitativo, de carácter descriptivo, del tipo relato de experiencia que se ha desarrollado en el contexto de una de las Unidades Básicas de Salud del municipio de Aracati, Ceará. La sistematización del relato se dio a partir de los registros de un diario de campo de la enfermera residente entre los meses de junio de 2013 y abril de 2015. Tras la implantación de la consulta de puericultura, se percibe la importancia de un seguimiento sistemático a partir del prenatal pasando por el momento del puerperio y alcanzando el foco principal de este estudio que es la salud del niño. Se destaca la contribución del Núcleo de Apoyo a la Salud de la Familia en las consultas y el uso de las tecnologías simples que favorezcan la interacción entre los profesionales, la comunidad y los servicios de salud. Además, algunas fragilidades del proceso de trabajo precisan ser superadas tales como los problemas estructurales, la falta de compromiso de algunos profesionales, las divergencias de conductas, la falta de motivación de los profesionales por la sobrecarga de trabajo y pocos cursos ofertados para la calificación del equipo de salud. Conclusión: La tarea no es fácil, los desafios son continuos pero la recompensa es mayor y más gratificante que cualquier dificultad vivida. Se espera que este relato promueva reflexiones críticas sobre el trabajo que se produce con la Estrategia de Salud de la Familia.

Descriptores: Atención Primaria de Salud; Salud del Niño; Enfermería Pediátrica; Comunicación Interdisciplinaria; Educación en Salud.

\section{INTRODUÇÃO}

A Atenção Primária à Saúde (APS) passou a ser estruturada a partir da Conferência Internacional sobre Cuidados Primários de Saúde, realizada em 1978, em Alma$\mathrm{Ata}^{(1)}$. Esse nível de atenção à saúde representa o primeiro contato dos indivíduos, da família e da comunidade com o sistema nacional de saúde, oferecendo serviços para que os usuários recebam atenção integral, tanto do ponto de vista do caráter biopsicossocial como através de ações de promoção, prevenção, cura e reabilitação, garantindo, portanto, a continuidade das ações de saúde e a longitudinalidade do cuidado $^{(2,3)}$.

A atenção à saúde da criança é considerada um campo prioritário quando se fala de saúde das populações ${ }^{(4)}$. Para que ela se desenvolvesse de forma mais eficaz, o Ministério da Saúde (MS) lançou, no ano de 1984, o Programa de Assistência Integral à Saúde da Criança (PAISC), com ações que visassem assegurar a integralidade da assistência e garantir que a criança desenvolvesse todo o seu potencial, com o intuito de reduzir a morbimortalidade na faixa etária de 0 a $5 \operatorname{anos}^{(5)}$.

Apesar de a mortalidade infantil mostrar uma tendência ao descenso após a intensificação desse programa e de outros, ainda se observava a permanência de elevadas taxas, nas quais a maioria das mortes infantis poderia ser evitada com medidas simples e eficazes. Implantou-se, no ano de 1997, a Estratégia Atenção Integrada às Doenças Prevalentes na Infância (AIDPI), com o objetivo de promover uma rápida e significativa redução da mortalidade, bem como a realização de ações integrais e o fortalecimento para que os problemas fossem resolvidos na APS ${ }^{(6)}$.

Essas iniciativas envolvendo a atenção à saúde da criança, bem como a ampliação dos serviços de saúde, foram potencializadas com a implantação da Estratégia Saúde da Família (ESF), considerada um marco na política de saúde brasileira pela proposta de reorganização e de operacionalizar a APS, ao assumir um modelo de atenção centrado na família e na equipe multiprofissional com acompanhamento longitudinal, com destaque para as ações destinadas à criança, no sentido de reduzir os agravos prevalentes nessa fase e potencializar a qualidade de vida dessa população $0^{(7)}$.

A consulta em puericultura tem como objetivo um acompanhamento criterioso do crescimento e desenvolvimento da criança pela equipe de saúde, e engloba um conjunto de medidas de cuidados preventivos, com um olhar abrangente que não só envolva a criança, mas também as condições em que a mãe e a família estão inseridas, adequando-se a consulta à realidade existente, buscando compreender as necessidades individuais ${ }^{(8)}$.

Esse acompanhamento é um importante instrumento para a promoção da saúde das crianças, a fim de garantir o crescimento e desenvolvimento adequados nos aspectos físico, emocional e social, contribuindo para a redução da morbidade e mortalidade infanti $1^{(9,10)}$. Assim, a integralidade na promoção da saúde passa a ser uma estratégia de produção de saúde que respeita as especificidades e potencialidades dos sujeitos, das coletividades e dos territórios, visando à equidade e à qualidade de vida, com redução de vulnerabilidades e riscos à saúde decorrentes dos determinantes sociais, econômicos, políticos, culturais e ambientais ${ }^{(11)}$.

Dessa maneira, para garantir a qualidade desse acompanhamento à criança, o MS recomenda sete consultas no primeiro ano de vida, além de duas consultas 
no segundo ano de vida e, a partir desta idade, consultas anuais $^{(8)}$. Destaca-se a assistência de enfermagem como sendo essencial nesse processo, uma vez que se tem como premissa atender integralmente a criança e estabelecer vínculos entre a família, o serviço e a equipe de saúde ${ }^{(12)}$.

Mediante a imersão na Unidade Básica de Saúde (UBS) do município de Aracati/CE, em que foi lotada enquanto enfermeira residente, a pesquisadora percebeu que as consultas de puericultura não eram realizadas, ou quando se concretizavam eram de maneira inadequada, reforçando o desconhecimento por parte das mães, familiares e comunidade acerca da importância do acompanhamento periódico e sistemático das crianças para avaliação de seu crescimento e desenvolvimento, sendo priorizado o cumprimento de práticas majoritariamente de caráter curativo.

A Residência Integrada em Saúde (RIS), conduzida pedagogicamente pela Escola de Saúde Pública do Ceará (ESP-CE), tem como uma de suas ênfases a Saúde da Família e Comunidade, cujo objetivo é "formar-ativar lideranças técnicas-científicas-políticas para qualificação da atenção primária visando a promoção, proteção e recuperação da saúde, embasada na colaboração interprofissional, na integralidade e na intersetorialidade, na perspectiva de contribuir para a consolidação da carreira na saúde pública e para o fortalecimento das Redes do Sistema Único de Saúde (SUS)"(13).

No contexto em que foi vivenciada a Residência, evidenciou-se a necessidade de implantação da consulta em puericultura e, através desta, a oportunidade de orientar as mães sobre as formas de cuidar do seu filho, acompanhar o crescimento e o desenvolvimento da criança gradualmente para que fosse possível a detecção precoce de agravos ou alguma alteração. Além disso, contou-se com a retaguarda do Núcleo de Apoio à Saúde da Família (NASF), para que as crianças acompanhadas pudessem se beneficiar de um cuidado integral e multiprofissional, com a ampliação da capacidade de cuidado da equipe in loco.

Diante do exposto, objetivou-se relatar a experiência da implantação da puericultura e desafios do cuidado na Estratégia Saúde da Família em um município do Estado do Ceará.

\section{SÍNTESE DOS DADOS}

Trata-se de um estudo de abordagem qualitativa, com caráter descritivo, do tipo relato de experiência, desenvolvido por uma enfermeira residente no contexto de uma das Unidades Básicas de Saúde (UBS) do município de Aracati, Ceará.

A referida UBS foi inaugurada em 2005, com sede própria e de acordo com os padrões preconizados pelo
Ministério da Saúde. Segundo o Sistema de Informação da Atenção Básica (SIAB), a Unidade em questão era responsável, em 2013, pelo acompanhamento de 1.573 famílias, com aproximadamente 5.778 pessoas $^{(14)}$. Existia uma área descoberta significativa calculada em 169 famílias, com aproximadamente 477 pessoas.

A equipe era composta por uma enfermeira, um médico, duas enfermeiras residentes, um cirurgião dentista, uma residente cirurgiã dentista, um auxiliar e um técnico de enfermagem, um auxiliar de farmácia, dois auxiliares de saúde bucal, seis agentes comunitários de saúde (ACS), um recepcionista, dois auxiliares de serviços gerais e uma equipe de NASF formada por um residente das categorias de Fisioterapia, Nutrição, Psicologia, Fonoaudiologia e Serviço Social do município que eram responsáveis por cinco áreas, incluindo a UBS supracitada.

A sistematização do relato se deu a partir dos registros realizados pela enfermeira residente, em um diário de campo, no período compreendido entre os meses de junho de 2013 e abril de 2015. Os registros fizeram referência à forma como se dava a participação e a interação das mães e os familiares durante as consultas e as contribuições dos atendimentos compartilhados com o NASF.

O momento após cada consulta era oportuno para que as anotações fossem registradas, mas devido a uma demanda expressiva, geralmente eram concretizadas ao final de um turno de atendimentos. Portanto, esses apontamentos eram realizados semanalmente a partir das observações e reflexões que se teciam sobre os atendimentos prestados.

\section{Conhecendo o território}

No primeiro contato com a UBS, foi realizada uma territorialização da área de abrangência como proposta metodológica do processo de formação da residência. A Política Nacional de Atenção Básica retrata a importância dos profissionais de saúde em participar no processo de territorialização e mapeamento da área de atuação da equipe, para que seja facilitada a identificação de grupos, famílias e indivíduos expostos a riscos e vulnerabilidades e, só assim, ofertar ações de acordo com as reais necessidades vigentes, potencializando maior resolutividade ${ }^{(15)}$.

Através da territorialização, verificou-se, quanto ao contexto histórico-turístico-cultural, que a comunidade apresentava uma identidade cultural forte, podendo também destacar-se como um local rico no que se refere às relações humanas. Já no quesito social, as vulnerabilidades do território, como o alto índice de uso e tráfico de drogas, alcoolismo, gravidez na adolescência e desemprego, abrangiam todos os aspectos da sociedade: cultural, financeiro, estrutural, entre outros, com hábitos e condições de vida que interferiam na saúde das pessoas. 
Após conhecer mais sobre a população adscrita e suas necessidades, e também mediante a imersão no território, foi evidenciado um cronograma de atendimentos que beneficiava somente alguns programas preconizados pelo MS e a uma demanda livre com um quantitativo expressivo. Foi percebida uma desorganização relacionada aos atendimentos no serviço de saúde e um total de ACS insuficiente para atender toda a área de abrangência. Evidenciou-se o cumprimento de práticas majoritariamente de caráter curativo e o desconhecimento por parte das mães, familiares e comunidade acerca da importância do acompanhamento periódico e sistemático voltado para a saúde da criança.

\section{Implantação das consultas de puericultura e fortalecimento do cuidado materno-infantil}

Ao detectar a fragilidade da realização da consulta em puericultura, buscou-se estabelecer um turno específico no cronograma para essa demanda - cada enfermeira da UBS tinha seu dia exclusivo e prioritário para esse atendimento -, como também divulgar essa informação para mães, familiares e comunidade em geral. Para tanto, enfatizou-se a oferta desse tipo de acompanhamento nas consultas de pré-natal e nas visitas puerperais, além de ampla divulgação pelos ACS, peças-chave das equipes de Saúde da Família, devido à sua importante inserção comunitária e seu potente agir comunicativo.

Durante as consultas de pré-natal, foram iniciadas as orientações relacionadas ao acompanhamento a ser realizado com as crianças. Nessa ocasião, foi possível conhecer um pouco do contexto social no qual a mãe e os familiares estavam inseridos, suas necessidades, bem como a história familiar e pessoal, com o intuito de identificar as prováveis condições de saúde da criança e fornecer às mães informações importantes relacionadas a seu futuro filho.

Após a assistência ao pré-natal, é essencial a visita domiciliar para as mães e seus filhos, mais habitualmente conhecida como visita puerperal, recomendada pelo MS na primeira semana pós-parto. Se não for possível nessa data, deve ser pactuada com a família a melhor forma de desenvolver esse acompanhamento em outro momento, para que não ocasione danos aos envolvidos ${ }^{(8)}$.

Durante as visitas domiciliares, conseguiu-se uma maior aproximação do contexto de vida daquelas famílias. Hábitos e crenças eram melhor compreendidos, as necessidades que permeavam a sua estrutura familiar, bem como os medos, a insegurança e os anseios da nova realidade. Nessa atuação, era de suma importância o olhar atento para a identificação de alguns sinais de alerta à saúde da criança e, também, um momento propício para a construção de vínculo, para que as mães e os familiares criassem confiança, tivessem maior segurança e participassem ativamente dos cuidados dispensados ao seu bebê.

A puericultura é uma estratégia utilizada na Saúde da Família e tem o papel de acompanhar o crescimento e o desenvolvimento infantil, a fim de promover uma melhor qualidade de vida, reduzir as incidências de doenças e aumentar as chances da criança desenvolver o seu potencial ${ }^{(9,16)}$. Cabe ao enfermeiro, responsável pela consulta, ofertar um cuidado humanizado, considerando a individualidade de cada criança e sua família e, através desse acompanhamento longitudinal, monitorar, avaliar e intervir de acordo com as necessidades identificadas ${ }^{(17)}$.

Durante a consulta da enfermagem com a criança, contemplava-se a anamnese e, em seguida, a avaliação do crescimento e do desenvolvimento por meio do exame físico céfalo-podálico, com mensuração de medidas antropométricas (peso, perímetro cefálico, perímetro torácico, estatura), além de avaliação da formação de dentição, ausculta cardíaca e pulmonar, marcos do desenvolvimento infantil, bem como a investigação de todo o estado geral da criança.

Além de pesar, medir e examinar a criança foi importante orientar as mães sobre ações de promoção da saúde, tais como: o incentivo ao aleitamento materno exclusivo até os seis meses de idade e, após esse período, introdução da alimentação complementar; banho de sol diariamente; higiene oral, íntima e ambiental; verificação do cartão vacinal; suplementação com o ferro após os seis meses, isto é, se não tivesse a necessidade antes; ensinar como estimular o filho no ambiente domiciliar; alguns exercícios de conforto em caso de cólicas; prevenção de acidentes no lar, dentre outros cuidados básicos de rotina. Destaca-se também a necessidade de conhecer o cotidiano da criança, a fim de se aprofundar e nortear a consulta.

A Política Nacional de Promoção da Saúde propõe que intervenções sejam ampliadas, para que as necessidades de saúde e seus determinantes e condicionantes sejam acolhidos com um olhar para além dos muros das unidades de saúde e do sistema de saúde, incidindo sobre as condições de vida e favorecendo uma ampliação de escolhas saudáveis ${ }^{(11)}$. Nesse sentido, a puericultura articula-se diretamente com os determinantes do processo saúde-doença e assume uma perspectiva ampliada de saúde, que, por sua vez, ressignifica a produção de cuidados.

O incentivo e o estímulo devem fazer parte do diálogo de todos os profissionais da saúde, para que os cuidadores (familiares ou outras pessoas) reconheçam seus esforços e capacidades, tornando-os mais confiantes nas suas habilidades e apoiados na dura tarefa de cuidar de uma criança $^{(18)}$. A promoção do cuidado deve ser desenvolvida através do diálogo, do acolhimento das necessidades 
individuais de cada mãe e seu filho e da interação com os profissionais de saúde, para que se construa um relacionamento harmonioso, usando-se de artifícios das tecnologias simples para estimular o aprendizado ${ }^{(19)}$.

Observou-se, através das consultas, que as mães sentiam a necessidade de serem escutadas em relação aos mais diversos assuntos que lhes causavam dúvida. A partir das suas próprias falas, foram construídas ações em conjunto, não impondo e ditando normas, inclusive para que estas não criassem restrições. É necessário utilizarse de atividades que contemplem a abordagem educativa, com linguagem coerente com a cultura de cada um, e não subestimar a forma de aprendizagem que o outro apresenta.

Para isso, a Educação em Saúde é uma prática que oportuniza a manifestação de medos e inseguranças, bem como fornece subsídios para uma autorreflexão dos enfermeiros e dos profissionais da saúde em geral de como estão desenvolvendo suas atividades. "É preciso ter cautela para que a educação em saúde não se transforme em um simples ato de depositar, transferir, de transmitir conhecimentos para as famílias (p. 396)"(20).

Ressalta-se a contribuição do NASF nas consultas, segundo a necessidade da avaliação ou do atendimento, sendo este realizado de forma compartilhada, compactuando-se em equipe como se daria esse suporte. As demandas eram as mais variadas possíveis, portanto, eram discutidos os casos e priorizados aqueles mais significativos.

Alguns casos foram selecionados quanto à necessidade da atuação multiprofissional: crianças com incoordenação de deglutição, dificuldade na mastigação, alterações nas curvas de crescimento (áreas de risco), atrasos de desenvolvimento psicomotor, comportamento alterado, casos de negligência, dentre outros. Esse formato de consulta foi a melhor configuração encontrada para que as crianças pudessem se beneficiar de um cuidado integral e que abrangesse todas as suas necessidades. Nessa perspectiva, o trabalho multiprofissional possibilita uma maior troca de saberes e experiências, estabelecendo a valorização no conhecimento do trabalho do outro, otimizando ações em conjunto, possibilitando maior resolubilidade em solucionar problemas individuais e coletivos demandados pela população e, com isso, proporcionando melhores serviços de saúde a comunidade ${ }^{(21)}$.

A cada relacionamento positivo, aumenta-se a adesão das mães às recomendações abordadas na consulta e, principalmente, o vínculo e a confiança com os profissionais de saúde, resultando em maior eficácia das ações de cuidado. $\mathrm{Na}$ área pediátrica, principalmente, é preciso que o profissional cultive a sensibilidade, promova o cuidado através da comunicação e da atenção individualizada, reconheça a mãe nas suas necessidades vigentes, saiba responder aos apelos surgidos ao desenvolver o cuidado na criança e consiga incluir e envolver a família nas práticas educativas. Portanto, é necessário acompanhar o que foi ensinado e tranquilizar a mãe, para que ela possa adquirir confiança em si e nos profissionais e retornar ao serviço para o acompanhamento do crescimento e desenvolvimento da criança $a^{(19)}$.

\section{Desafios enfrentados no processo de trabalho para sustentabilidade do cuidado longitudinal à criança}

A consulta em puericultura foi implantada na referida UBS com o objetivo de acompanhar o crescimento e o desenvolvimento das crianças, visando à promoção da saúde e prevenção de agravos. Porém, para que ela fosse concretizada, foi preciso superar alguns obstáculos do processo de trabalho.

Os obstáculos estruturais observados foram quantidade de salas e profissionais insuficientes, falta de insumos e equipamentos (antropômetro infantil, balança ideal, fita métrica, materiais socioeducativos, dentre outros) e pouca disponibilidade de transporte, prejudicando a realização das visitas domiciliares. Com relação à atuação da residente na UBS foram percebidas dificuldades como a falta de compromisso de alguns profissionais; divergências de condutas; profissionais desmotivados devido à sobrecarga de trabalho e poucos cursos ofertados para qualificação da equipe de saúde. Perceberam-se, também, limitações referentes ao apoio da gestão do município.

Ao vivenciar a realização dessa prática, ficou mais fácil compreender que nem sempre as condutas indicadas irão levar aos resultados esperados e que as mães e/ou os familiares, algumas vezes, não seguem as orientações concedidas e, provavelmente, conduzem a situação da maneira mais conveniente e adequada para sua situação. Portanto, é preciso cultivar a sensibilidade para perceber quando as intervenções não estão sendo eficazes e rever as metodologias empregadas.

Destaca-se que o uso dos dispositivos e as tecnologias simples estão interligados, pois não se constrói uma assistência humanizada sem relacioná-los, ou seja, não há vínculo sem um bom acolhimento, como também não há corresponsabilização e autonomia sem vínculo. A escolha por essa metodologia nas práticas de saúde é essencial para que se alcance a sustentabilidade do cuidado longitudinal e a humanização do cuidado.

O trabalho em saúde deve ser construído em conjunto. Apesar das especificidades e particularidades de cada profissão, quando ele é realizado de forma multiprofissional resulta em melhor qualidade na assistência, ampliando o cuidado e a integração de conhecimentos referentes às formas de cuidar. Assim, grandes entendimentos são 
proporcionados às mães, filhos, familiares e aos próprios profissionais da saúde.

Desse modo, a criatividade é uma peça fundamental do enfermeiro durante o exercício de sua profissão, para que possa reinventar e trabalhar com o que lhe é ofertado. Mesmo diante de um contexto desafiador, os resultados foram os mais gratificantes possíveis retratados através da valorização do trabalho. As reflexões acerca das práticas de saúde produzidas tornam-se imprescindíveis para desenvolver uma autocrítica e rever se as tecnologias utilizadas estão refletindo de maneira positiva na assistência à saúde da comunidade.

\section{CONCLUSÃO}

A partir deste relato, torna-se necessário destacar a inovação que a residência multiprofissional em saúde traz para os profissionais em geral, para o município que recebe esses trabalhadores da saúde e para a comunidade, pois oferta uma sucessão de aprendizados e de como realmente se fazer saúde. Este programa contempla as necessidades locais, regionais e nacionais de saúde, integrando saberes e práticas que permitem construir novas competências, habilidades e atitudes, trazendo, portanto, transformações, melhorias e contribuindo para uma melhor qualidade de vida destinada à população.

O processo de trabalho voltado para a saúde maternoinfantil envolvia a atenção ao pré-natal e cuidados no puerpério por meio de visitas domiciliares, com o intuito de fortalecer o vínculo com a mãe e sensibilizá-la acerca da importância das consultas de puericultura. Investiu-se nas ações de promoção da saúde, orientações diversas quanto aos cuidados gerais, incentivo ao empoderamento dos sujeitos envolvidos e estímulo a uma maior aproximação com o serviço na perspectiva da saúde, e não somente diante de processos de adoecimento. Para tanto, o fortalecimento do vínculo entre profissionais de saúde e as famílias sob sua responsabilidade sanitária possibilitou relações de confiança e respeito, percebendo-se uma maior aceitação dos aprendizados proporcionados durante as consultas.

O enfermeiro, profissional cujo objetivo do trabalho é o cuidado, deve se instrumentalizar de estratégias simples e resolutivas, lançar mão do uso do acolhimento, diálogo, vínculo, corresponsabilização e escuta ativa entre profissional e usuário do serviço de saúde, para que, com isso, seja possível ofertar um atendimento pautado na integralidade. Além disso, as atividades realizadas em conjunto com o NASF, as decisões tomadas em equipe, as experiências compartilhadas, a troca de saberes e práticas conseguiram elucidar mais ainda a importância de se trabalhar multiprofissionalmente, refletindo em resultados concretos observados nas vivências domiciliares.
A tarefa não é fácil, os desafios são contínuos, mas a recompensa consegue ser maior e mais gratificante do que qualquer dificuldade vivenciada. Acredita-se que trabalhar de forma humanizada ainda é a forma mais admirável de se proporcionar saúde para uma comunidade em condição de vulnerabilidade. Espera-se que este relato promova reflexões críticas acerca do trabalho que se tem produzido em saúde.

\section{REFERÊNCIAS}

1. Baratieri T, Marcon SS. Longitudinalidade do cuidado: compreensão dos enfermeiros que atuam na Estratégia Saúde da Família. Esc Anna Nery Rev Enferm. 2011;15(4):802-10.

2. Starfield B. Atenção primária: equilíbrio entre necessidades de saúde, serviços e tecnologia. Brasília: Unesco; 2002.

3. Silva SA, Fracolli LA. Avaliação da assistência à criança na Estratégia de Saúde da Família. Rev Bras Enferm. 2016;69(1):47-53.

4. Machado MMT, Lima ASS, Bezerra JG Filho, Machado MFAS, Lindsay AC, Magalhães FB et al. Características dos atendimentos e satisfação das mães com a assistência prestada na atenção básica a menores de 5 anos em Fortaleza, Ceará. Ciênc Saúde Coletiva. 2012;17(11):3125-33.

5. Ministério da Saúde (BR). Assistência integral à saúde da criança: ações básicas. Brasília: Ministério da Saúde; 1984.

6. Ministério da Saúde (BR), Organização Mundial da Saúde. Organização Pan-Americana da Saúde. AIDPI Atenção Integrada às Doenças Prevalentes na Infância: curso de capacitação. $2^{a}$ ed. Brasília: Ministério da Saúde; 2002.

7. Reichert APS, Leônico ABA, Toso BRG, Santos NCCB, Vaz EMC, Collet N. Orientação familiar e comunitária na Atenção Primária à Saúde. Ciênc Saúde Coletiva. 2016;21(1):119-27.

8. Ministério da Saúde (BR), Secretaria de Atenção à Saúde, Departamento de Atenção Básica. Saúde da criança: crescimento e desenvolvimento. Brasília: Ministério da Saúde; 2012. (Cadernos de Atenção Básica, $\left.n^{\circ} 33\right)$.

9. Vasconcelos VM, Frota MA, Martins MC, Machado MMT. Puericultura em Enfermagem e educação em saúde: percepção de mães na Estratégia Saúde da Família. Esc Anna Nery Rev Enferm. 2012;16(2): 326-31. 
10. Malaquias TSM, Gaíva MAM, Higarashi IH. Percepções dos familiares de crianças sobre a consulta de puericultura na estratégia saúde da família. Rev. Gaúch Enferm. 2015;36(1):62-8.

11. Ministério da Saúde (BR), Secretaria de Vigilância à Saúde, Secretaria de Atenção à Saúde. Política Nacional de Promoção da Saúde: PNaPS: revisão da Portaria MS/GM n 687, de 30 de março de 2006. Brasília: Ministério da Saúde; 2014.

12. Britto PF. Seguimento do recém-nascido de alto risco: um desafio à equipe de saúde e de enfermagem [dissertação]. Rio de Janeiro: Universidade do Estado do Rio de Janeiro; 2006.

13. Escola de Saúde Pública do Ceará - ESP-CE, Secretaria da Saúde do Estado do Ceará. Edital n ${ }^{\circ}$ 03/2013 [acesso em 2015 Mar 19]. Disponível em: http://www. esp.ce.gov.br/index.php?option=com_content\&view $=$ article\&id=2775: edital032013

14. Ministério da Saúde (BR), DATASUS. Sistema de Informação da Atenção Básica- SIAB. Ceará [Internet]. [acesso em 2015 Mar 19]. Disponível em: http://www2. datasus.gov.br/SIAB/index.php?area $=04$

15. Ministério da Saúde (BR), Secretaria de Atenção à Saúde, Departamento de Atenção Básica. Política Nacional de Atenção Básica. Brasilia: Ministério da Saúde; 2012. (Série E. Legislação em Saúde).

16. Campos RMC, Ribeiro CA, Silva CV, Saparolli ECI. Consulta de Enfermagem em puericultura: a vivência do Enfermeiro na Estratégia de Saúde da Família. Rev Esc Enferm USP. 2011;45(3):566-74.
17. Gautério DP, Irala DA, Cezar-Vaz MR. Puericultura em Enfermagem: perfil e principais problemas encontrados em crianças menores de um ano. Rev Bras Enferm. 2012;65(3):508-13

18. Veríssimo MDLOR. Conversando sobre cuidar de crianças. In: Secretaria Municipal da Saúde de São Paulo, Coordenação do Programa Saúde da Família. Toda hora é hora de cuidar: manual de apoio. $1^{\mathrm{a}}$ ed. São Paulo; 2003. p.48-51.

19. Queiroz MV, Jorge MS. Estratégias de educação em saúde e a qualidade do cuidar e ensinar em Pediatria: a interação, o vínculo e a confiança no discurso dos profissionais. Interface Comun Saúde Educ. 2006;10(19):117-30.

20. Góes FGB, La Cava AM. A concepção de educação em saúde do enfermeiro no cuidado à criança hospitalizada. Rev Eletrônica Enferm [internet]. 2009 [acesso em 2015 Abr 08];11(4):932-41. [acesso em 2015 Abr 08]. Disponível em: https://www.fen.ufg.br/fen_revista/ v11/n4/pdf/v11n4a19.pdf

21. Costa JP, Jorge MSB, Vasconcelos MGF, Paula ML, Bezerra IC. Resolubilidade do cuidado na atenção primária: articulação multiprofissional e rede de serviços. Saúde Debate. 2014;38(103):733-43.

\section{Endereço para correspondência:}

Dellane Giffoni Soares

Universidade Estadual do Ceará - UECE

Curso de Enfermagem

Av. Dr. Silas Munguba, 1700

Bairro: Campus do Itaperi

CEP 60740-000 - Fortaleza - CE - Brasil

E-mail: delanegiffoni@hotmail.com 